periods. Migrant workers are a particularly healthy group from within their home countries and if they develop cancer, it is likely to occur when they are older and have left Qatar. The impact of occupational exposures on cancer risk among industrial workers in Qatar can therefore not be estimated. It is however possible to measure exposures in these settings and establish adequate national regulations. Information on carcinogenic exposures in occupational settings from the European CAREX database is likely of limited use in Qatar and other Middle Eastern countries, due to use of different materials, processes and technologies.

\section{BREAST CANCER INCIDENCE AMONG FLIGHT ATTENDANTS}

${ }^{1}$ Mary Schubauer-Berigan, ${ }^{1}$ Misty Hein, 'Jeri Anderson, ${ }^{2}$ Steven Allee, ${ }^{3}$ Alice Sigurdson, ${ }^{3}$ Mark Little, 'Lynne Pinkerton. ${ }^{1}$ National Institute for Occupational Safety and Health, Centers for Disease Control and Prevention, Cincinnati, OH, USA; ${ }^{2}$ SRA International, Inc., Fairfax, VA, USA; ${ }^{3}$ National Cancer Institute, National Institutes of Health, Bethesda, MD, USA

\subsection{6/oemed-2014-102362.142}

Objectives Previous studies suggest that flight attendants have a higher incidence of breast cancer than the general population; however, the reason remains unclear. We evaluated the relation of breast cancer incidence with estimates of cosmic radiation dose and metrics of circadian rhythm disruption among a cohort of 6092 female former US flight attendants.

Method Cohort members (or their proxy) completed a computer assisted telephone interview that collected data on incident cancers and non-occupational risk factors for breast cancer. Incident cancers were also identified through linkage with state cancer registries. Life table analyses were conducted to compare breast cancer incidence among the cohort to that in the general population and to evaluate exposure-response relations.

Results Breast cancer incidence was increased compared to the general population (observed 343; standardised incidence ratio 1.37; 95\% confidence interval 1.23, 1.52). Among flight attendants, breast cancer was not significantly associated with ten-year lagged cumulative estimates of absorbed cosmic radiation dose, time spent working during normal sleep hours, or time zones crossed in the cohort overall or in women who gave birth two or fewer times. A significant positive association was observed between breast cancer incidence and these exposures only in the small subset of women who gave birth three or more times.

Conclusions Our data suggest that the effect of occupational exposures on breast cancer risk among flight attendants may be modified by non-occupational risk factors for breast cancer. Future modelling analyses will further evaluate the role of occupational exposures and non-occupational risk factors.

\section{THE RELATIONSHIP BETWEEN WELDING FUME EXPOSURE AND CHRONIC OBSTRUCTIVE PULMONARY DISEASE IN SHIPYARD WELDERS IN KOREA}

${ }^{1}$ Dong-Hee Koh, ${ }^{2}$ Jung-II Kim, ${ }^{3}$ Kun-Hyung Kim, ${ }^{4}$ Seung-Won Yoo. ${ }^{1}$ Carcinogenic Hazard Brach, National Cancer Control Institute, National Cancer Center, Goyang-Si, GyeonggiDo, Republic of Korea; ${ }^{2}$ Dong-a University, Busan, Republic of Korea; ${ }^{3}$ Department of Occupational and Environmental Medicine, Busan Paik Hospital, Inje University, Busan, Republic of Korea; ${ }^{4}$ Korean Industrial Health Association, Suwon, Republic of Korea ${ }^{5}$ Occupational Safety and Health Research Institute, Korea Occupational Safety and Health Agency, Incheon, Republic of Korea

10.1136/oemed-2014-102362.143
Objectives Welding fume is suspected to accelerate the decline of lung function and development of chronic obstructive pulmonary disease (COPD). The aim of this study was to examine the relationship between welding fume exposure and COPD in Korean shipyard welders.

Method 240 male welders who were working at two shipyards and took the annual health examination including pulmonary function test in 2010 participated in this study. A questionnaire about smoking habits and occupational history was administered. PFT was carried out with strict quality control measures. Exposed fume concentrations were estimated using 884 welding fume measurements taken 2002-2009 in one of the shipyards. Linear multiple regression was employed to evaluate the association between cumulative fume exposure and lung function parameters. Logistic regression was employed to test the excess risk of COPD by cumulative fume exposure. Age, height, the smoking amount, and cumulative fume exposure were incorporated as independent variables in those models.

Results Mean age was 48, and mean work duration was 18 years. The mean cumulative fume exposure was $7.7 \mathrm{mg} / \mathrm{m}^{3}$. The prevalence of COPD was $14.6 \% . \mathrm{FEV}_{1}$ and FVC showed negative correlations with cumulative fume exposure, but statistically non-significant. Odds ratios of COPD were significantly elevated for middle (5.02, 95\% CI:1.27-33.55) and high exposure group (6.20, 95\% CI:1.41-44.98) compared to the low fume exposure group.

Conclusions Our findings suggest a potential association between metal fume exposure and COPD. Further study with a prospective design is needed to investigate the excessive decline of lung function by welding fume exposure.

\section{POOLING CASE-CONTROL STUDIES FOR ENHANCED EVIDENCE ON OCCUPATIONAL RISK FACTORS IN LUNG CANCER RESEARCH - THE SYNERGY PROJECT}

1,2 Ann Olsson, ${ }^{3}$ Hans Kromhout, ${ }^{3}$ Roel Vermeulen, ${ }^{3}$ Susan Peters, ${ }^{4}$ Beate Pesch, ${ }^{4}$ Thomas Behrens, ${ }^{4}$ Benjamin Kendzia, ${ }^{1} J o a c h i m ~ S c h u ̈ z, ~{ }^{1}$ Kurt Straif. ${ }^{1}$ International Agency for Research on Cancer, Lyon, France; ${ }^{2}$ The Institute of Environmental Medicine, Karolinska Institutet, Stockholm, Sweden; ${ }^{3}$ Institute for Risk Assessment Sciences, Utrecht, The Netherlands; ${ }^{4}$ Institute for Prevention and Occupational Medicine of the German Social Accident Insurance - Institute of the Ruhr-Universität Bochum, Bochum, Germany

10.1136/oemed-2014-102362.144

Objectives Explore quantitative exposure-response association for exposure to asbestos, crystalline silica, nickel, chromium and polycyclic aromatic hydrocarbons in the general population; further study effects on specific cell types and potential interaction with smoking and co-occurring occupational exposures.

Method Fourteen studies from Europe and Canada were pooled including 17700 lung cancer cases and 21800 controls with detailed information on tobacco habits and lifetime occupations. A quantitative job-exposure-matrix (SYN-JEM) was developed based on more than 350.000 exposure measurements from the participating countries. Different model specifications were compared to predict historical job-, time-, and region-specific exposure levels. Individual exposure levels were calculated for each subject by linking the SYN-JEM with the individual occupational histories. Unconditional logistic regression models were fitted to estimate odds ratios, 95\% confidence intervals, and trends.

Results We observed exposure-response relationships with increasing duration and cumulative exposure for all agents and 
generally saw a stronger effect for squamous- and small cell lung carcinomas than for adenocarcinomas. Smoking and simultaneous exposure to other occupational exposures exerted a minor confounding effect on the risk estimates. The effect modifications with smoking tended to be supra-additive.

Conclusions SYNERGY adds valuable knowledge to the field of occupational cancer epidemiology, and underlines the importance to collect data on histology, and lifelong information on occupational exposures and smoking.

\section{EXPOSURE-RESPONSE ESTIMATES FOR DIESEL ENGINE EXHAUST AND LUNG CANCER MORTALITY BASED ON DATA FROM THREE OCCUPATIONAL COHORTS}

${ }^{1}$ Roel Vermeulen, ${ }^{2}$ Debra Silverman, ${ }^{3}$ Eric Garshick, ' 'Jelle Vlaanderen, 'Lützen Portengen, ${ }^{4}$ Kyle Steenland. 'IRAS Utrecht University, Utrecht, The Netherlands; ${ }^{2}$ National Cancer Institute, Bethesda, MD, USA; ${ }^{3}$ Harvard Medical School, Boston, MA, USA; ${ }^{4}$ Emory University, Atlanta, GA, USA

\subsection{6/oemed-2014-102362.145}

Objectives To derive a meta-exposure-response curve (ERC) for DEE and lung cancer mortality and estimate lifetime excess risks (ELRs) of lung cancer mortality based on assumed occupational and environmental exposure scenarios.

Method We conducted a meta-regression of lung cancer mortality and cumulative exposure to elemental carbon (EC), a proxy measure of DEE, based on relative risk (RR) estimates reported by three large occupational cohort studies. Based on the derived risk function, we calculated ELRs for several lifetime occupational and environmental exposure scenarios, and also calculated the fractions of annual lung cancer deaths attributable to DEE.

Results We estimated a lnRR of 0.00098 (95\% CI: 0.00055 , $0.0014)$ for lung cancer mortality with each $1-\mu \mathrm{g} / \mathrm{m}$ 3-year increase in cumulative EC. Estimated numbers of excess lung cancer deaths through age 80 for lifetime occupational exposures of 1,10 , and $25 \mu \mathrm{g} / \mathrm{m} 3 \mathrm{EC}$ were 17, 200, and 689 per 10000 , respectively. For lifetime environmental exposure to $0.8 \mu \mathrm{g} / \mathrm{m} 3$ EC, we estimated 21 excess lung cancer deaths per 10000 . Based on broad assumptions regarding past exposures we estimate that approximately $6 \%$ of annual lung cancer deaths may be due to DEE exposure.

Conclusions Combined data from three US occupational cohort studies suggest that DEE at levels common in the workplace and in outdoor air appear to pose substantial excess lifetime risks of lung cancer, above usually acceptable limits in the US and Europe, which are generally set at 1/1000 and 1/100 000 based on lifetime exposure for the occupational and general population, respectively.

\section{MORTALITY OF A COHORT OF WORKERS IN GREAT BRITAIN WITH BLOOD LEAD MEASUREMENTS}

${ }^{1}$ Damien McElvenny, ${ }^{1}$ Brian Miller, ${ }^{1}$ Laura MacCalman, 'Anne Sleeuwenhoek, ${ }^{1}$ Martie van Tongeren, ${ }^{2}$ Kevin Shepherd, ${ }^{2}$ Andrew Darnton, ${ }^{1}$ John Cherrie. ${ }^{1}$ Institute of Occupational Medicine, Edinburgh, UK; ${ }^{2}$ Health and Safety Executive, Bootle, UK

\subsection{6/oemed-2014-102362.146}

Objectives We report on the first ever analysis of a UK cohort of workers with blood lead level measurements that was assembled in the late 1970s.
Method As an alternative to mean and maximum blood lead levels, we carried out an exposure assessment that assigned workers to high, medium or low exposure to lead. We additionally assessed whether workers would be exposed to an important level of relevant co-carcinogens.

Results 3466 deaths were observed among 7770 men and 1352 women. The SMRs for all causes $(109,95 \%$ CI 105-112) and all malignant neoplasms (113,107-120) were significantly raised. SMRs for oesophageal, stomach, bladder, brain and kidney cancer and non-malignant kidney disease were not raised, but were raised for lung cancer (142,129-157). The SMR for circulatory diseases $(105,99-100)$ was of borderline significance. No trends were observed for mean or maximum blood lead level or assessed lead exposure for any of the cancers of a priori interest, but a significant association was found for circulatory diseases (ischaemic heart disease) with mean and maximum blood lead level.

Conclusions The excess of lung cancer is possibly to be due to tobacco smoking. This study provides strong evidence to support an association between increased lead exposure and increased risk of ischaemic heart disease mortality. The study is, however, limited by the lack of complete occupational histories for the included participants.

\section{OCCUPATIONAL EXPOSURE TO CARCINOGENS IN NEW ZEALAND}

Andrea't Mannetje, David McLean, Bill Glass, Kerry Cheung, Jeroen Douwes, Neil Pearce. Centre for Public Health Research, Massey University, Wellington, New Zealand

\subsection{6/oemed-2014-102362.147}

Objectives To provide a detailed assessment of the extent and industrial distribution of workplace carcinogens in New Zealand, and to identify key industries and key carcinogens for which intervention would result in marked reductions in occupational cancer.

Method A literature review was conducted identifying the carcinogens present in and the cancer risks associated with each respective industry. A New Zealand specific Information System on Occupational Exposure to Carcinogens (NZ-CAREX) was developed.

Results There are more than 50 known human carcinogens commonly present in New Zealand workplaces. The most common of these are: asbestos; benzene; chromium VI compounds; formaldehyde; involuntary smoking; occupational exposures as a painter; silica; solar radiation; wood dust. The industries for which an increased cancer risk has been observed repeatedly in epidemiological studies and have the highest number of potentially exposed workers include: agriculture; construction; health services; machinery and equipment manufacturing; metal product manufacturing; and wood/paper product manufacturing. Of the agricultural chemicals currently in use in New Zealand's agricultural sector, none are known human carcinogens, but numerous are suspected to be carcinogenic to humans. A number of barriers for the implementation of intervention strategies were identified, which could be removed by improving access to knowledge regarding the occurrence of carcinogens in New Zealand workplaces and improving access to control tools and methods to evaluate their effectiveness.

Conclusions Exposure to carcinogens remains common in New Zealand workplaces, and many opportunities remain for reducing exposure and the associated occupational cancer burden. 